\title{
ABERROMETRY FROM ASTRONOMY TO VISION SCIENCE
}

\author{
Type of article: Hypothesis and Idea \\ Nassima Ould Amer ${ }^{1}$, Fatiha Kail ${ }^{1}$, Mohammed Amine Derdour ${ }^{2}$, Selma Chiali ${ }^{1}$, Mohammed El \\ Amine Lazouni ${ }^{3}$, Larabi Chahed ${ }^{1}$ \\ ${ }^{1}$ LPCMME, Faculty of Exact and Applied Sciences, University of Oran1, Algeria \\ ${ }^{2}$ Cogneau University Hospital Establishment, Faculty of Medicine, University d'Oran1, Algeria \\ ${ }^{3}$ Abou Bakr Belkaid University of Tlemcen, Algeria
}

\begin{abstract}
Aberrometry is a branch of optical metrology and more specifically of astronomy. This branch is applied to the science of vision. It allows dramatic improvements in human vision research, such as refining the visual capabilities of healthy and diseased subjects beyond physiological limits.

Aberrometry relies on the ocular wave front collection with the help of an aberrometer, which allows establishing the precise and quantitative record of the various optical aberrations of the human eye, whether low or high degree.

In this work, we draw our approach to apply these concepts to refractive surgery and adaptation in order to relate the changes in Higher-Order Aberrations (HOAs) following wavefront-guided femtosecond laser-assisted (WFG FS-LASIK), and to explore the correlations between preoperative spherical equivalence (SE) and mechanisms of HOAs affecting the visual quality.

Keywords: Aberrometry, adaptive optics, ocular aberrations, wavefront, refractive surgery.

Corresponding author: Ould Amer Nassima, LPCMME, Facultés des sciences exactes et appliquées, Université d’Oran1, Algeria.nassimaouldamer23@gmail.com

Received: 20 December, 2018, Accepted: 01 June, 2019, English editing: 04 July, 2019, Published: 13 July, 2019.

Screened by iThenticate..(2017-2019 KNOWLEDGE KINGDOM PUBLISHING.
\end{abstract}

\section{Introduction}

The eye corresponds to an optical system with some optical elements to focus rays of light onto the retina to make up an image. Imperfections present in the eye constituent materials may deviate light rays from the intended path, aka optical or Wavefront Aberrations (WAs). Since WAs complicate light focusing by the retina, they cause defects in the perceived image that have, as end results, blurred pictures and declined visual exactitude. There are two types of WAs:

a) Lower order aberrations (LOAs): They are another way to refer to refractive errors: myopia, hyperopia in addition to astigmatism. All of these pathologies can be corrected by glasses, contact lenses or Laser (refractive surgery). LOAs are modeled by second-order Zernike polynomials that characterize the common aberrations' defocus, e.g., hyperopia, myopia, and astigmatism. LOAs account for nearly $85 \%$ [15] of all eye aberrations.

b) Higher order aberrations (HOAs): They consist of optical imperfections that cannot be fixed by any trustworthy equipment or technology. Practically everybody has at least some degree of HOAs that can be diagnosed and measured now through technological advances like the wavefront aberrometer. HOAs correspond to aberrations whose Zernike polynomials have expressions higher than second-order terms as is the case with the third-order Zernike terms that appear in coma and trefoil. Fourth-order Zernike terms correspond to spherical aberrations, and so on. HOAs account for $15 \%$ of all WAs. 
This work is concerned with HOAs that were first measured in humans by Smirnov in 1961 using a psychophysical method that predicted HOA compensation by customized lenses [1]. An improved technique was developed by Howland [2] that measured WAs of the human eye objectively, using a Hartmann-Shack sensor in 1994 [3]. After some HOAs had been efficiently alleviated with adaptive optics, which improved the optical quality of healthy eyes [4], the ability to provide supernormal vision and high-resolution retinal imaging [5] attracted a great deal of attention.

For conventional refractive surgery, the high degree optical aberration rate increased, essentially for the coma-type and spherical aberrations. Wavefrontguided refractive surgery is a personalized treatment that makes drastic changes and reductions of pre-existing HOAs, and prevents additional HOAs. It is a reliable technique, which is becoming within reach of mainstream treatment. Wavefront guided refractive surgery has recently been introduced in Algeria, and it is something the visual health care professionals are yet to embark upon beaucause no studies have been performed. It is the main reason for our investigation, as well as evaluating the impact of this kind of surgery on visual performances of Algerian myopic eyes.

The Hartmann-Shack aberrometer is used to determine the individual Zernike coefficients from third-up to sixth-order and the root mean square of total higherorder aberrations, and to determine the suitable pupil diameter for the surgery. In addition, it allows the collection of the pre and postoperative HOAs after six months. In this study, we evaluate and compare the changes in the HOAs subsequent wavefront-guided laser-assisted femtosecond, and investigate the correlations between preoperative spherical equivalence and components of the HOAs affecting visual quality.

A retrospective study was performed on patients suffering from myopia referred to Tlemecen Clinic Northern Algeria, a Hartmann-Shack aberrometer was used to determine the individual Zernike coefficients from the third- to the sixth order, as well as the mean square of the total aberrations of the higher order, and anticipate the appropriate pupil diameter for surgery. On the other hand, the aberrometer makes it possible to collect HOA before and after surgery, i.e. after six months. In this study, we evaluated and compared changes in HOA after femtosecond laserguided front and laser-guided assistance, and examined the correlations between preoperative spherical equivalence and HOA components affecting visual quality. To do that, we have divided this paper as follows: in section two, we present our proposed methods. In section three, we present the aberrations' Zernike polynomials representation. In section four, a brief description of aberrometry in vision science. In section five, we present the potential outcomes of our proposed method. Finally, we conclude the paper.

\section{The proposed method}

Currently, our retrospective study is made on a database composed of twenty myopic eyes examined by means of the aberrometry measurements at Lazouni Clinic, a private clinic in Tlemcen, western Algeria. 
Nine myopic eyes from five patients were enrolled in the study retrospectively after having undergone WFG FS-LASIK.

- Uncorrected distance visual acuity (UDVA)

- Corrected distance visual acuity (CDVA)

- Refractive error

- Individual Zernike coefficients of 3rd- to 6th-order HOAs were measured before and 3 months after the surgeries and were compared using the Mann-Whitney test or Student's t-test.

\section{Modeling of the aberrations Zernike polynomials representation}

The WA analyses aim at evaluating the optical eye quality by measuring the shape of WAs. For this, aberrometer equipment or a wavefront sensor can determine the different corneal wavefront aberrations. There exists three different categories of aberrometers:

(i) The outgoing wavefront aberrometer such as the Hartmann-Shack sensor [6];

(ii) The ingoing retinal imaging aberrometer such as the cross-cylinder aberrometer [7], Tscherning aberrometer [8] and the sequential retinal ray tracing method

(iii) The ingoing feedback aberrometer employed in the spatially resolved refractometer [9] and the optical path difference scheme [10]

The wavefront shape can be analyzed using an expansion of it into sets of Zernike polynomials (ZPs). The ZPs are a combination of independent trigonometric functions that can be combined to describe WAs appropriately because of their orthogonality. Fig. 1 depicts Zernike polynomials ranging from the first to sixth orders.
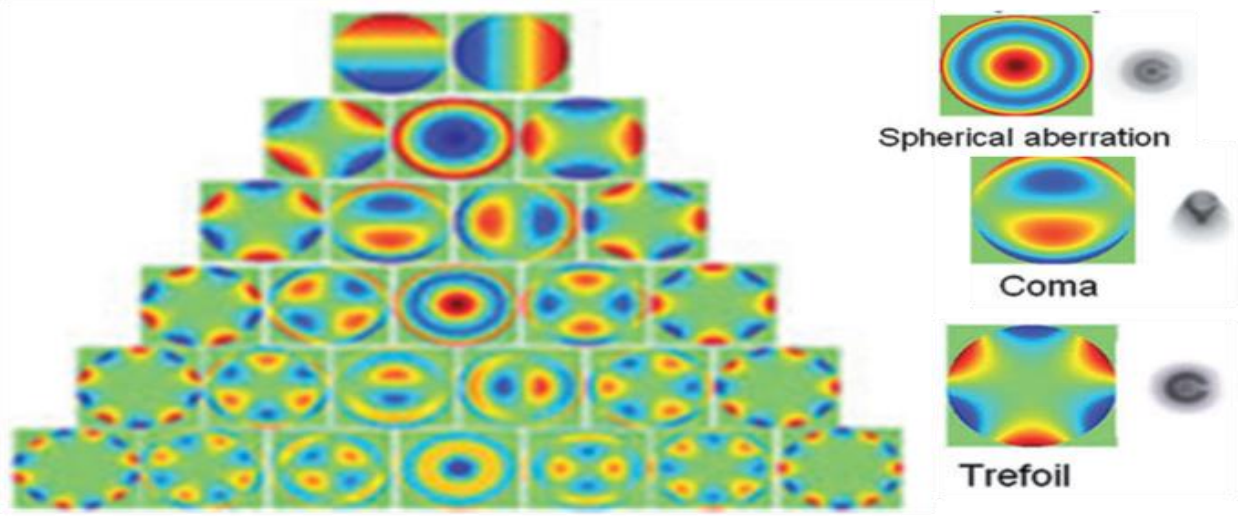

Figure.1. Graphical representation of the six orders Zernike polynomials [8].

\section{Application in vision science}

\subsection{Wavefront-guided refractive surgery}

The Aberrometry examination is essential before a refractive surgery (PRK or LASIK). 
This examination provides the eye aberrations of the patient in order to construct a personalised treatment using the excimer laser for custom-made ablation [17].

The signs of progress in wavefront analyses allow the growth detection of the eye HOAs with subsequent conventional photorefractive keratectomy (PRK) [10, 11].

Thus, customized ablation to correct irregular astigmatism or to lessen the surgically generated irregular astigmatism might resolve some of the problems induced by the conventional keratorefractive methods. Still, there exist several factors to be solved to augment the effects of wavefront-guided refractive surgery, such as wavefront registration errors between states at measurement and treatment, unpredictable corneal shape alterations due to cicatrization and/or biomechanics, HOA fluctuations, beam profile variations, and so on.

\section{2. Contact lens}

Aberrometry during the time a subject wears contact lenses and spectacles can introduce differences in the HOAs.

Different studies in the state of the art have revealed lower HOAs with RGP lenses than with soft contact lenses or spectacles [12].

There was an increase in the total number of HOAs during the period of wearing soft contact lenses for myopia problems compared with those without contact lenses [13].

The techniques used to manufacture the contact lenses [14] can impact the differences found in the HOAs

\section{Tne obtained results: Application to the Algerian cornea}

The advantages of laser refractive operation with the flying spot excimer laser system have been acknowledged and the smoother surface is one of the paramount influences on the visual aftermath. Due to the small ablation area, the energy power is additionally uniform, with less shock wave, and can be effortlessly controlled. After the laser procedure, there is practically no corneal surface step. The smoother surface allows faster visual recovery, with better UCVA and gains Best-Corrected Distance Visual Acuity (BCDVA), which is a parameter used in cataract surgery.

Wavefront-guided LASIK, which was established to rectify HOAs, has been reported as being better than standard LASIK and one of the finest corneal refractive surgery technologies. Nevertheless, the postoperative regression or overcorrection risks have been reported to grow in human subjects with elevated myopia or astigmatism.

Wavefront-guided LASIK has been accomplished by the excimer laser system to apply a flying spot with a Gaussian profile and active eye tracker. The iris pattern image taken preoperatively with the wavefront aberrometer was the input to the ablation using iris-recognition software. All patients were seated, with the quantity of cyclorotation adjusted immediately before the laser ablation. A standard profile (non-wavefront) ablation corrected the sphere and refractive cylinder to achieve emmetropia. 
Aberrometric measurements have been performed on patients that underwent refractive surgery in the Lazouni private clinic, Tlemcen. Changes in HOAs from pre and postoperative conditions in six clinical cases pointed towards the reduction in HOAs, but the rate and the specificity of the Algerian cornea could not be determined for these preliminary results because the number of patients is limited. For Keratoconus, which is an eye disorder that progressively thins the cornea, further

Studies are planned at the Ibn EL Haythem Centrer for contact Lens Adaptation, Algiers, to analyze the changes in the rate of the HOAs on patients with Keratoconus before and after wearing rigid contact lenses, and to compare the finding with previous studies previous studies.

The authors need to develop databases with multimodal images for future experiments as conceived in other medical areas [21, 22, and 23]. As more experiments are conducted with these eye images, better simulations and analyses will be done. These databases will also help when it comes to more advanced diagnoses using Content-Based Image Retrieval (CBIR), and better eye 3D models can be developed with the help of super-resolution [18, 19, 20, and 23].

\section{Conclusions}

Aberrometry is an optical metrology branch and it can be applied to ophthalmology. It allows dramatic ameliorations in human vision, e.g., refining the visual abilities of human subjects beyond physiological bounds. It permits observing with very high resolution the photoreceptors in retinal imageries for earlier diagnosis of some retinal pathologies as well as the scrutiny and assessment of better functioning treatments. They rely on the ophthalmic wavefront gathering with an aberrometer that permits forming the precise quantitative record of the various human eye aberrations for LOA and HOA. This work proposes an approach to apply these models to refractive surgery and contact lenses adaptation for the case of HOAs following WFG FS-LASIK, and to analyze the correlations concerning preoperative SE and the mechanisms of HOAs affecting visual quality.

\section{Conflict of interest statement}

We certify that there is no conflict of interest with any financial organization in the subject matter or materials discussed in this manuscript.

\section{Authors' biography}

Nassima OULD AMER is a PHD student at university Oran1, Oran, Algeria. She received her master degree in optometry from the University of Oran 1 in 2012.

Fatiha Kail is a Professor at university Oran1, Oran, Algeria. She received the Ph.D degree in Material Sciences from the University of Reims, Reims, France, in 2005. She worked as researcher at PICM lab of Ecole Polytechnique, in Palaiseau, France, from 2002 to 2006 and at Girona and Barcelona Universities, from 2008 to 2013. In 2014, She rejoined the Department of Physic, University oran1, as associated professor, and becomes a Professor in 2016. Her current research interests include Materials Sciences, Optics and Optometry. Pr. Kail is a chair of optics and 
optometry group of PCMME Lab, university Oran1. She is an expert member of Conference Régionale des Universities de l'Ouest (CRUO).

DERDOUR MOHAMMED AMINE is an ophthalmologist Professor at Hamou Boutlélis ophthalmology hospital in Oran. He is especially interested in research in machine learning, data mining, image processing, medical and biologic data classification and intelligent decision support systems. He is a member of the research laboratory: "Laboratoire de génétique médicale". He is interested in several ophthalmic research projects.

Larbi Chahed is Professor at university Oran1, Oran, Algeria. He received the Ph.D degree in solid state Physics from Oran university, in1989. Since, he becomes professor, head of PCMME Lab of Oran university, head of Physics department and rector of Oran and SBA universities. Actually, he is General Director of Higher Education and Training at M.E.S.R.S. His research interests are on Materials Sciences and Optics.

Mohammed El Amine LAZOUNI is an Assistant professor at Abou Bekr Belkaid Tlemcen University, Algeria. He received his master degree in Electronics Biomedical from Tlemcen University in 2010. In 2014, he obtained his PhD Thesis in the Biomedical Engineering Laboratory of Abou Bekr Belkaid Tlemcen University. He is especially interested in research in machine learning, data mining, image processing, medical and biologic data classification and intelligent decision support systems.

Selma Chiali is associated Professor at university Oran1, Oran, Algeria. She received the Ph.D degree in Physics from Oran university, in1993. She taught physics and optics in physics department during 35 years. She is senior researcher in in optometry group of PCMME Lab, University Oran1 Her research interests are on Optics and optometry. In 2016, she becomes head of Institut des Sciences et Techniques Appliquées (ISTA).

\section{References}

[1].Tyson RK. History and background. In: Principles of Adaptive Optics, 2nd edn. Boston, MA: Academic Press, 1998; 1-25.

[2]. Howland B, Howland HC. Subjective measurement of high-order aberrations of the eye. Science 1976; 193: 580-2. https://doi.org/10.1126/science.959814 PMid:959814

[3]. Liang J, Grimm B, Goelz S, Bille JF. Objective measurement of wave aberrations of the human eye with the use of a Hartmann-Shack wave-front sensor. J Opt Soc Am A 1994; 11: 1949-57. https://doi.org/10.1364/JOSAA.11.001949

[4]. Liang J, Williams DR, Miller DT. Supernormal vision and high-resolution retinal imaging through adaptive optics. J Opt Soc Am 1997; A14: 2884-92 https://doi.org/10.1364/JOSAA.14.002884

[5]. Roorda A, Williams DR. The arrangement of the three cone classes in the living human eye. Nature 199397: 520-2. https://doi.org/10.1038/17383 PMid:10028967

[6]. Thibos LN. Principles of Hartmann-Shack aberrometry. J Refract Surg 2000; 16: S563-65.

[7] . Mrochen M, Kaemmerer M, Mierdel P, Krinke HE, Seiler T. Principles of Tscherning aberrometry. J Refract Surg 2000; 16: S570-1.

[8] Molebny VV, Panagopoulou SI, Molebny SV, Wakil Y, Pallikaris IG. Principles of ray tracing aberrometry. J Refract Surg 2000; 16: S572-575.

[9] . Burns SA. The spatially resolved refractometer. J Refract Surg 2000; 16: S566 - 9. https://doi.org/10.3928/1081-597X-20000901-15

[10]. Mierdel P, Kaemmerer M, Krinke HE, Seiler T. Effects of photorefractive keratectomy and cataract surgery on ocular optical errors of higher order.

[11]. Seiler T, Kaemmerer M, Mierdel P, Krinke HE.Ocular optical aberrations after photorefractive keratectomy for myopia and myopic 13 Schallhorn SC, Farjo AA, Huang $\mathrm{D}$ et al., American Academy of Ophthalmology. Wavefrontguided LASIK for the correction of primary myopia and astigmatism: a report by the American Academy of Ophthalmology. Ophthalmology 2008, 115: 1249-61.

[12]. Mihashi T, Hirohara Y, Koh S, Ninomiya S, Maeda N, Fujikado T. Tear film break-up time evaluated byreal-time Hartmann-Shack wavefront sensing. Jpn JOphthalmol 2006; 50: 85-9.58. Koh S, Maeda N, Hirohara Y et al. Serial measurements of higher-order 
aberrations after blinking in normal subjects. Invest Ophthalmol Vis Sci 2006; 47:3318-24. https://doi.org/10.1167/iovs.06-0018 PMid:16877397

[13]. Koh S,Maeda N, Hori Y et al. Effects of suppression of blinking on quality of vision in borderline cases ofevaporative dry eye. Cornea 2008; 27: 275-8. https://doi.org/10.1097/ICO.0b013e31815be9c8 PMid:18362651

[14]. Montes-Mico R, Caliz A, Alio JL. Wavefront analysis of higher order aberrations in dry eye patients. J Refract Surg 2004; 20: 243-7.

[15] Resan M, Vukosavljević M, Milivojević M. Wavefront Aberrations, Advances in Ophthalmology, Shimon Rumelt, IntechOpen, 2012, https://doi.org/10.5772/24441 PMCid:PMC3384963

[16] Maeda PY Zernike Polynomials and their Use in describing the Wavefront Aberrations of the human Eye, Stanford University, <http://www. stanford. edu/ pmaeda>(2003).

[17] Berlien, H.-P. "Principles of Laser Application in Medicine". Medical Technologies Journal, Vol. 2, no. 3, Sept. 2018, pp. 228-9, doi:https://doi.org/10.26415/2572-004Xvol2iss3p228-229. https://doi.org/10.26415/2572-004X-vol2iss3p228-229

[18] de Jesus, M.A., Estrela, V.V., Saotome, O., \& Stutz, D. (2018) Super-resolution via particle swarm optimization variants. In: Hemanth J., Balas V. (Eds). Lecture Notes in Comp. Vision and Biomechanics, vol 25. Springer, Cham. https://doi.org/10.1007/978-3319-61316-1_14

[19] Fernandes, S.R., Estrela, V.V., Magalhaes, H.A., \& Saotome, O. (2014) On improving sub-pixel accuracy by means of B-Spline, In Proc. of the 2014 IEEE Int'l Conf. on Imaging System and Techniques (IST 2014), Santorini, Greece. doi: 10.1109/IST.2014.6958448 PMid:24266705

[20] Herrmann, A. E. \& Estrela, V. V. (2016). Content-based image retrieval (CBIR) in remote clinical diagnosis and healthcare. In M. Cruz-Cunha, I. Miranda, R. Martinho, \& R. Rijo (Eds.), Encyclopedia of E-Health and Telemedicine (pp. 495-520). Hershey, PA: IGI Global. doi:10.4018/978-1-4666-9978-6.ch039 https://doi.org/10.4018/978-1-4666-9978$6 . \operatorname{ch} 039$

[21] Mahmoodi, M. S., and S. A. Mahmoodi. "Design of CAD System of Solitary Pulmonary Nodule With Harmony Classification and Fuzzy System". Medical Technologies Journal, Vol. 1, no. 4, Nov. 2017, pp. 102-, doi: 10.26415/2572-004X-vol1iss4p102-102.

[22] Gozali, E., B. Rahimi, M. Sadeghi, and R. Safdari. "Diagnosis of Diseases Using Data Mining". Medical Technologies Journal, Vol. 1, no. 4, Nov. 2017, pp. 120-1, doi: 10.26415/2572-004X-vol1iss4p120-121

[23] Jesus, M.A., \& Estrela, V.V. (2017). An Introduction to Data Mining Applied to HealthOriented Databases, Orient. J. Comp. Sci. and Technol (OJCST), 9(3). DOI : https://doi.org/10.13005/ojcst/09.03.03 